\title{
Response rate and varied reinforcement: Reinforcers of different strengths ${ }^{1}$
}

\author{
WARREN M. STEINMAN ${ }^{2}$ \\ UNIVERSITY OF ILLINOIS
}

Twelve rats were trained to lever press on a multiple schedule with the delivery of pellets, sucrose, or pellets or sucrose correlated with the three legs of the schedule. The "pellet-orsucrose" condition, i.e., varied reinforcement, produced the highest response rates. These results replicate, in a within-Ss design, the previous findings of a between-group experiment.

In a previous experiment (Steinman, 1966), groups of rats were trained to lever press under four different reinforcement conditions. For three groups, the type of reinforcer delivered was always the same within groups but different between groups. A fourth group received varied reinforcement, i.e., one or another of the three reinforcers was delivered on a given reinforcement occasion but the particular reinforcer delivered varied randomly from occasion to occasion. The varied-reinforcement group developed a much higher response rate than was found in any of the constant-reinforcement groups.

The present experiment attempts to replicate the essential character of the above study using a withinSs design rather than a between-groups design. In addition, the experiment investigates the variedreinforcement effect when the reinforcers comprising the variety are clearly different in reinforcing strength. Subjects

Twelve experimentally naive male hooded rats of the Long-Evans strain served as Ss. When about 100 days of age they were placed in individual cages and maintained on a food deprivation cycle permitting access to Purina Lab Chow for $1 \mathrm{~h}$ per day. Water was arailable ad lib.

\section{Apparafus}

Two sound-restricted chambers were housed in separate rooms. Each chamber contained a $13.5 \mathrm{x}$ 7.5 in. Skinner box with a 2 in. wide metal lever on one wall and a $3.5 \times 3$ in. panel on the opposite wall. Behind the panel was an area for the delivery of $45 \mathrm{mg}$ food pellets and a fountain device for the delivery of sucrose solution. A $7 \mathrm{~W}$ indirect house light provided the only source of constant illumination. All programming and recording equipment was automatic and inaudible to $\mathrm{S}$.

\section{Procedure}

Ss were trained to press the lever, which on a variable interval schedule having a mean interval of $45 \mathrm{sec}$ (VI 45) resulted in the onset of a $0.5 \mathrm{sec}$ indicator light. Pushing the panel after the onset of the light produced a $45 \mathrm{mg}$ food pellet.
Phase 1. When the above behavioral chain had been established, a tone $\left(T_{1}\right)$ of either 400,1900 , or 4000 cps was presented for six 5 min periods within each session. Completed chains during $T_{1}$ were reinforced with food pellets. At the end of a 5 min period, the tone ceased and no further responding was reinforced. This time out from reinforcement (TO) continued until $S$ refrained from lever pressing or panel pushing for a minimum of $15 \mathrm{sec}$, at which time $T_{1}$ was reinstated. When for three consecutive sessions the lever pressing rates during $T_{1}$ appeared to be asymptotic and no TO lasted more than $20 \mathrm{sec}$, Phase 1 was concluded.

Phase 2. A second tone $\left(\mathrm{T}_{2}\right)$ was added in Phase 2. During $\mathrm{T}_{2}$ responding was reinforced with a $.07 \mathrm{cc}$ drop of $30 \%$ sucrose solution. Five minute periods of $T_{1}$ were randomly followed by 5 min periods of $\mathrm{T}_{2}$ within each session. Between each tone was the TO condition. Again, a session consisted of six 5 min periods.

Phase 3. When lever pressing had stabilized during each of the two tones and responding during TO was again minimal, a third 5 min tone $\left(T_{3}\right)$ was added. During $T_{3}$ the reinforcer to be delivered on a particular reinforcement occasion varied randomly between the food pellets and sucrose solution. The 5 min tone periods were also randomly scheduled and each tone period was preceded by a TO. A session was composed of nine tone presentations.

Results

The combined performance of $\mathrm{Ss}$ in each session of Phase 3 is illustrated in Fig. 1. As in the research cited above, the sucrose solution produced a higher

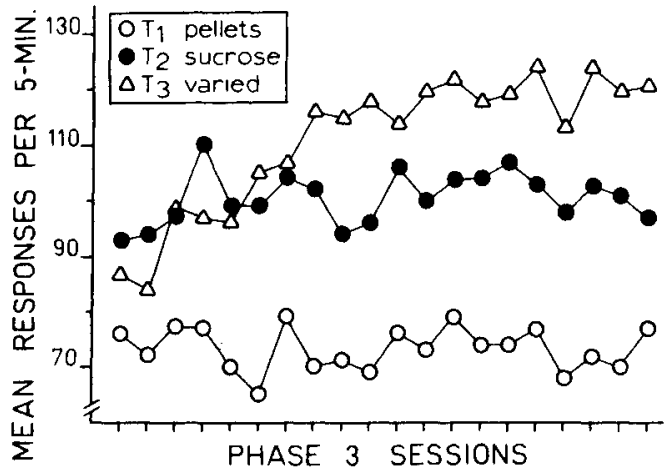

Fig. 1. Mean lever presses per 5-min in each session of Phase 3. 
response rate than did the food pellets. Furthermore, the rate during the varied-reinforcement condition $\left(\mathrm{T}_{3}\right)$ was higher than in either of the other conditions $\left(T_{1}\right.$ and $\left.T_{2}\right)$. These differences were found to be statistically reliable $(F=8.09, p<.01)$. A Duncan's test (Edwards, 1960) indicated that each of the reinforcement conditions produced reliably different rates $(p<.01)$.

All $12 \mathrm{Ss}$ responded at a higher rate in $\mathrm{T}_{2}$ than in $T_{1}$. Nine Ss also responded consistently at a higher rate during $T_{3}$ than during $T_{2}$. For two $S s$ the higher rate during $T_{3}$ did not evolve until approximately half way through Phase 3 . The final $S$ performed during $T_{3}$ at a rate grossly equivalent to that of $T_{2}$.

\section{Discussion}

Previous research has tended to emphasize the similarities between varied-reinforcement and partial-reinforcement schedules (e.g., Logan, 1960; Wunderlich, 1961; cf., Steinman, 1966). To the extent that the reinforcers comprising the varied-reinforcement condition differ in reinforcing strength, a situation is developed which has partial reinforcement as its limit.

Although a partial-reinforcement analysis may be useful in explaining the greater resistance to extinction of a response acquired and maintained by varied reinforcement, it does not seem to be similarly useful in explaining the higher response rates produced by varied reinforcement in the present experiment. It is important to note that in all three reinforcement conditions, Ss received reinforcement on a VI schedule. In the varied-reinforcement condition, the strongest of the two reinforcers (sucrose) was delivered only half as frequently as during the "sucrose-only" condition. Thus, there was what amounted to a longer average interval between equally strong reinforcers in the varied-reinforcement condition as compared with the sucrose-only condition. Variable interval schedules which have comparatively longer mean intervals, i.e., lower reinforcement densities, tend to produce lower response rates than when the reinforcement density is higher (Ferster \& Skinner, 1957). The fact that the rates were higher under the varied-reinforcement condition than under the sucrose-only condition suggests that something other than the differential reinforcing strengths of the two reinforcers produced the varied-reinforcement effect.

\section{References}

EDWARDS, A. L. Experimental Design in Psychological Research. New York: Rinehart \& Co., 1960.

FERSTER, C. B., \& SKINNER, B. F. Schedules of Reinforcement. New York: Appleton-Century-Crofts, 1957.

LOGAN, F. A. Incentive. New Haven: Yale University Press, 1960.

STEINMAN, W. M. Generalized conditioned reinforcement under a single deprivation condition. Psychol Rec., 1966, 16, 457-472.

WUNDERLICH, R. A. Strength of a generalized conditioned reinforcer as a function of variability of reward. J. exp. Psychol, 1961, $409-415$.

Notes

1. This research was supported by Grant No. 13111-01 from the National Institutes of Mental Health.

2. Address: Children's Research Center, University of Illinois, Champain, Illinois, 61820 .

\section{Erratum}

Bowen, J., \& McCain, G. Occurrence of the partial reinforcement extinction effect after only one NRNR sequence of trials. Psychon. Sci., 1967, 9, 15-16: On page 15, paragraph 3 , the authors erroneously assumed that Surridge et al used the same extinction confinement $(90 \mathrm{sec})$ in Experiment II as they had in Experiment I. The confinement time actually used was 30-35 sec. 